\title{
Association of retinal venular tortuosity with impaired renal function in the Northern Ireland Cohort for the Longitudinal Study of Ageing
}

\author{
R. A. O’Neill, A. P. Maxwell, F. Kee, I. Young, B. McGuinness, R. E. Hogg and McKay GJ
}

\begin{abstract}
Background: Previous studies have identified retinal microvascular features associated with renal dysfunction. Biopsies are necessary to confirm kidney microvascular damage and retinal imaging may enable evaluation of microangiopathic characteristics reflecting renal changes associated with chronic kidney disease (CKD). We evaluated retinal microvascular parameters (RMPs) for associations with renal function in a cross-sectional analysis of the Northern Ireland Cohort for the Longitudinal Study of Ageing.

Methods: RMPs (central retinal arteriolar/ venular equivalents [CRAE/CRVE], arteriolar to venular ratio [AVR], fractal dimension and tortuosity) were measured from optic disc centred fundus images using semi-automated software. Associations were assessed with multivariable regression analyses between RMPs and estimated glomerular filtration rate (eGFR) defined by serum creatinine (eGFRscr) and cystatin C (eGFRcys) and also CKD status characterised by eGFR $<60 \mathrm{~mL} / \mathrm{min} / 1.73 \mathrm{~m}^{2}$. Regression models were adjusted for potential confounders including age, sex, diabetes, smoking status, educational attainment, cardiovascular disease, body mass index, antihypertensive medication, systolic blood pressure, triglycerides, high- and low-density lipoprotein levels.
\end{abstract}

Results: Data were included for 1860 participants that had measures of renal function and retinal fundus images of sufficient quality for analysis. Participants had a mean age of $62.0 \pm 8.5 \mathrm{yrs}$. and $53 \%$ were female. The mean eGFR for scr and cys were $82.2 \pm 14.9 \mathrm{~mL} / \mathrm{min} / 1.73 \mathrm{~m}^{2}$ and $70.7 \pm 18.6 \mathrm{~mL} / \mathrm{min} / 1.73 \mathrm{~m}^{2}$ respectively. eGFRcys provided lower estimates than eGFRscr resulting in a greater proportion of participants categorised as having CKD stages 3-5 (eGFRcys 26.8\%; eGFRscr 7.9\%). Multivariable regression analyses showed that increased venular tortuosity (OR= 1.30; 95\%Cl: 1.10, 1.54; $P<0.01$ ) was associated with CKD stages $3-5$ characterised by eGFRscr $<60 \mathrm{~mL} / \mathrm{min} / 1.73 \mathrm{~m}^{2}$. No additional associations between CKD status characterised by eGFRscr or with eGFRcys, were detected $(P>0.05)$. Multivariable regression failed to detect associations between CRAE, CRVE, AVR, fractal dimension or tortuosity and eGFRscr or eGFRcys $(P>0.05)$.

Conclusion: Increased retinal venular tortuosity was associated with CKD stages 3-5 defined by eGFRscr $<60 \mathrm{~mL} /$ $\mathrm{min} / 1.73 \mathrm{~m}^{2}$, in an older population independent of potential confounding factors. These retinal measures may provide non-invasive microvascular assessment of associations with CKD.

Keywords: Retinal microvascular parameters, Renal function, Chronic kidney disease, Estimated glomerular filtration rate

\footnotetext{
* Correspondence: g.j.mckay@qub.ac.uk

Centre for Public Health, Queens University Belfast, Belfast, UK
}

C C The Author(s). 2020 Open Access This article is licensed under a Creative Commons Attribution 4.0 International License, which permits use, sharing, adaptation, distribution and reproduction in any medium or format, as long as you give appropriate credit to the original author(s) and the source, provide a link to the Creative Commons licence, and indicate if changes were made. The images or other third party material in this article are included in the article's Creative Commons licence, unless indicated otherwise in a credit line to the material. If material is not included in the article's Creative Commons licence and your intended use is not permitted by statutory regulation or exceeds the permitted use, you will need to obtain permission directly from the copyright holder. To view a copy of this licence, visit http://creativecommons.org/licenses/by/4.0/. The Creative Commons Public Domain Dedication waiver (http://creativecommons.org/publicdomain/zero/1.0/) applies to the data made available in this article, unless otherwise stated in a credit line to the data. 


\section{Background}

Global increases in ageing have been widely reported and those $>50$ years (yrs) represent the most rapidly expanding demographic in the Northern Ireland population [1]. Social economic development and heath care improvements have increased life expectancy impacting upon societal health care systems, policies and demands [2-4]. Chronic kidney disease (CKD) is a major global health concern with estimates suggesting between 3 and $18 \%$ of the population are affected, [1, 2] leading to substantial economic burden $[3,4]$ and diminished quality of life [5]. CKD incidence and prevalence is greatest among the elderly [3] and is expected to increase further over the coming decades as populations age [6]. CKD is characterised by irreversible reductions in the excretory and homeostatic functions of the kidneys [7] leading to a higher risk of adverse outcomes including cardiovascular mortality [8], and is predicted to become the fifth most common cause of death worldwide by 2040 [9]. Improved non-invasive, early-stage kidney disease detection would offer clinical utility for the identification of individuals at increased risk of CKD for targeted intervention to limit the extent and rate of kidney function loss [10].

Common biomarkers of renal function and damage include serum creatinine, cystatin $C$, and proteinuria but their ability to identify those at greatest risk of future decline is limited [1]. Several biomarkers have improved CKD detection and risk prediction [11] and while tissuederived markers have proven useful for the identification of accumulated renal microvascular damage [12-16], they tend to be less amenable to non-invasive assessment [17].

Microvascular pathology is commonly found in eye and kidney diseases with several studies reporting associations between renal impairment and retinal microvascular variation although the findings have not always been consistent [12-16]. Such associations may be indicative of systemic vascular effects and renovascular damage [18]. Furthermore, similarities in the cellular physiological characteristics that characterise the renal and retinal microcirculation, including retinal pericytes and renal mesangial cells, implicate similar pathological pathways leading to end organ damage [19]. Advances in retinal imaging modalities and analysis applications readily identify microangiopathic variation in the eye. These innovations provide novel opportunities to assess if specific retinal anatomical features can be correlated with measures of kidney function $[17,20,21]$. As such, the aim of this study was to assess retinal microvascular parameters (RMPs) in association with baseline measures of renal function in a cross-sectional analysis of older persons from the Northern Ireland Cohort for the Longitudinal Study of Ageing (NICOLA).

\section{Methods \\ Study characteristics}

NICOLA is a longitudinal cohort study of 8468 community dwelling men and women aged 50 years and over, resident in Northern Ireland (individuals in care homes or other residential institutions were excluded at baseline) [22]. The study, established in 2012, has three main components: a computer aided personal interview (CAPI), a self-completion questionnaire and health assessment (Supplementary file 1). The CAPI was extensive in scope and included assessment of demographic, social and health-related factors, and was conducted at individual appointments in each participant's home between December 2013 and March 2016. Measures of cardiovascular, physical, cognitive and visual function were determined and a biobank of biological samples collected simultaneously which included visual health with retinal fundus photography. Written informed consent was obtained from participants prior to participation under ethical approval from the School of Medicine, Dentistry and Biomedical Sciences Ethics Committee, Queen's University Belfast (SREC 12/23) and in accordance with the Helsinki Declaration.

\section{Measurement of renal function and classification of CKD}

Serum creatinine (scr, $\mathrm{mg} / \mathrm{dL}$ ) standardised to isotope dilution mass spectrometry (IDMS) calibrated techniques and cystatin $\mathrm{C}$ (cys, mg/L) were assayed on an Abbott ARCHITECT c8000 system using kinetic alkaline picrate and turbidimetric/ immunoturbidimetric methods, respectively. The coefficient of variation for creatinine and cystatin $\mathrm{C}$ was $<4.68$ and $<1.80 \%$ respectively. Estimated glomerular filtration rate (eGFRscr and eGFRcys) was based on a single serum sample using the Chronic Kidney Disease Epidemiology Collaboration equation (CKD-EPI 2009 equation for scr and 2012 equation for cys) $[23,24]$. CKD stages $3-5$ were defined as eGFR $<60 \mathrm{~mL} / \mathrm{min} / 1.73 \mathrm{~m}^{2}$ and CKD stages $1-2$ as $\mathrm{eGFR} \geq 60 \mathrm{~mL} / \mathrm{min} / 1.73 \mathrm{~m}^{2}$.

\section{Other variables}

Systolic blood pressure (SBP) was calculated as the average of two individual measurements. Diabetes status was characterised as a combination of $\mathrm{HbA} 1 \mathrm{c} \geq 6.5 \%$, use of diabetic medications and self-reported diabetes at CAPI and health assessment. Educational attainment was dichotomised: primary and lower or secondary level and above (including university education). Smoking status was categorised as current smokers versus non-smokers. Cardiovascular disease (CVD) was by self-report and characterised by a history of angina, heart attack, 
congenital heart failure or stroke. Participants were excluded if scr or cys were missing or retinal images were of insufficient quality for image analysis (Fig. 1).

\section{Retinal image acquisition and measurement}

Retinal photography was performed through the dilated pupil using a Canon CX-1 Digital Fundus Camera (Canon USA, Melville, NY, USA), following dilation from a single drop of $1 \%$ tropicamide in all participants. RMPs (central retinal arteriolar/ venular equivalents [CRAE/CRVE], arteriolar to venular ratio [AVR], fractal dimension and tortuosity) were measured from optic disc centred fundus images, collected at participant health assessment and analysed using the semi-automated software Vessel Assessment and Measurement Platform for Images of the Retina (VAMP IRE; VAMPIRE group, University of Dundee, Dundee, Scotland, Version 3.1; Fig. 2), by a qualified grader blinded to participant data $[25,26]$. Images were taken from the left eye except when unavailable or of poor quality, in which case the right eye image was used. Intraclass correlation coefficients (ICCs) were calculated to assess intergrader reliability with mean values of 0.87 (CRAE) and 0.91 (CRVE).

\section{Statistical analysis}

All analyses were performed using Statistical Package for Social Sciences (Version 24.0. Armonk, NY: IBM Corp).

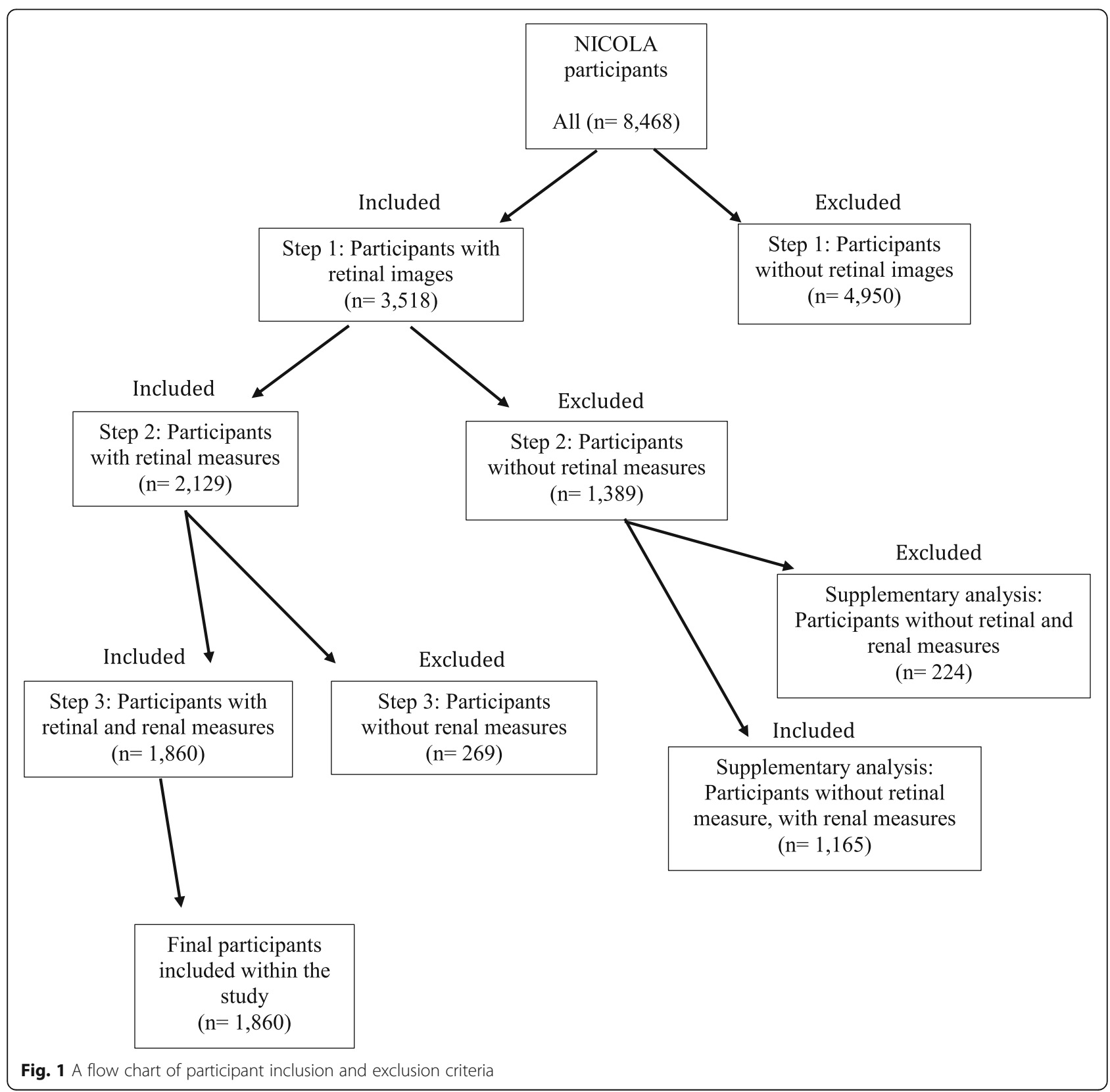




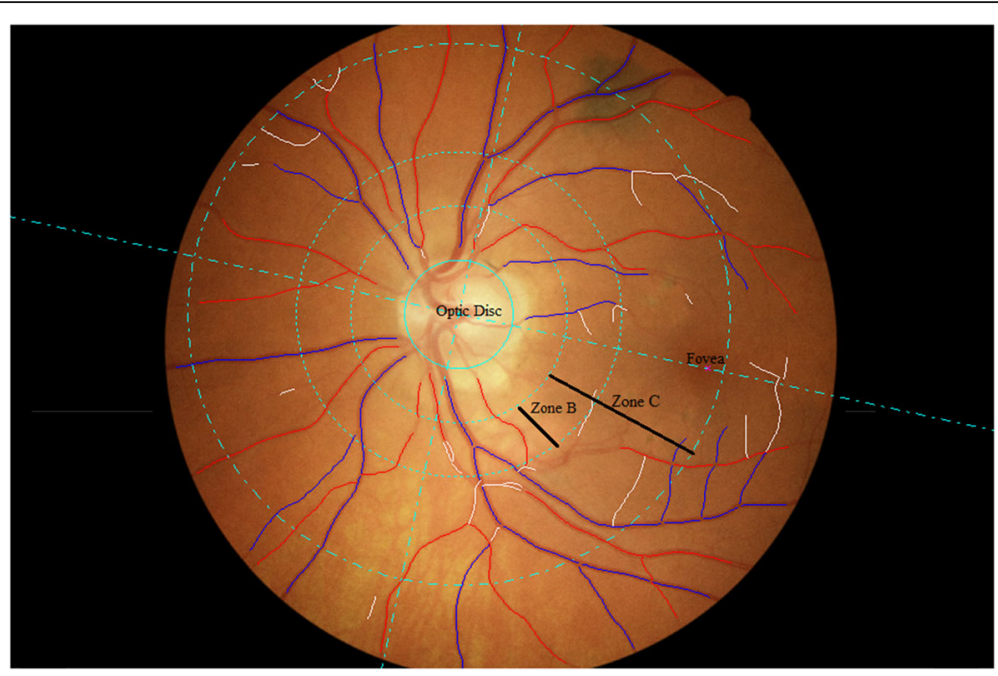

Fig. 2 Optic disc centred retinal fundus image assessed using the Vessel Assessment and Measurement Platform for Images of the Retina (VAMP IRE) software. Arterioles (red), venules (blue) and deleted segments (white) are indicated. The retinal microvascular parameters for arteriolar and venular calibre (CRAE, CRVE, and AVR), fractal dimension and tortuosity are calculated from measurements captured in zones B and C (1.0 to 2.5 optic disc diameters from the disc margin)

Before inclusion in regression models all RMPs were transformed into standardised Z-scores. Independent samples t-tests and chi-squared tests were used to compare the distribution of demographic factors and clinical variables between participants characterised as CKD stages 1-2 and CKD stages 3-5. Population characteristics were described using mean and standard deviation (SD) for continuous variables or frequencies and percentages for categorical variables. Linear and logistic regression were used to evaluate associations between RMPs and renal function (eGFRscr and eGFRcys) and the binary trait of CKD status. Minimally adjusted models included age and sex with fully adjusted models also including diabetes and smoking status, educational attainment, CVD, body mass index (BMI), antihypertensive medication, systolic blood pressure, triglycerides, high and low-density lipoprotein (HDL and LDL) levels. $P<0.05$ was considered statistically significant.

\section{Results}

From 8468 participants, data was available for 1860 participants with measures of renal function and retinal fundus images of sufficient quality (Fig. 1). Participant characteristics are summarised in Table 1; comparisons between participants with retinal fundus images included with in the study or not, are summarised in Supplementary Table 1. A STROBE Statement (STrengthening the Reporting of OBservational studies in Epidemiology) is a reporting guideline that includes a checklist of 22 items considered essential for good reporting of observational studies and is presented in Supplementary Table 2. Participants had a mean age of $62.0 \pm 8.5$ yrs. and $53 \%$ were female. The mean eGFR for scr and cys were $82.2 \pm 14.9$ $\mathrm{mL} / \mathrm{min} / 1.73 \mathrm{~m}^{2}$ and $70.7 \pm 18.6 \mathrm{~mL} / \mathrm{min} / 1.73 \mathrm{~m}^{2}$ respectively. SBP was $131 \pm 18.5 \mathrm{mmHg}$ and $23 \%$ of participants had diabetes mellitus. There were 147 participants with CKD stages $3-5$ characterised by eGFRscr $<60 \mathrm{~mL} / \mathrm{min} /$ $1.73 \mathrm{~m}^{2}$ compared to 498 using eGFRcys (CKD stages $3-$ $5=7.9 \%$ vs $26.8 \%$ respectively). As expected, participants with CKD stages 3-5 had significantly lower mean eGFR compared to participants with CKD stages 1-2 (eGFRscr: $49.6 \pm 7.8 \mathrm{~mL} / \mathrm{min} / 1.73 \mathrm{~m}^{2}$ versus $85.0 \pm 11.7$ $\mathrm{mL} / \mathrm{min} / 1.73 \mathrm{~m}^{2}$, and eGFRcys: $49.1 \pm 8.0 \mathrm{~mL} / \mathrm{min} / 1.73$ $\mathrm{m}^{2}$ versus $78.6 \pm 14.7 \mathrm{~mL} / \mathrm{min} / 1.73 \mathrm{~m}^{2}$ ). Participants with CKD stages $3-5$ had a significantly higher mean age compared to participants with CKD stages 1-2 (eGFRscr: $70.5 \pm 9.0$ yrs. versus $61.3 \pm 8.1$ yrs. and eGFRcys: $68.2 \pm 8.3$ yrs. versus $59.8 \pm 7.4$ yrs). The difference between eGFRscr and eGFRcys estimates against mean eGFR, is presented in a Bland Altman plot (Supplementary Figure 1). A lower percentage of participants in CKD stages 3-5 were female than CKD stages 1-2 (eGFRscr: $44 \%$ versus $54 \%$ and eGFRcys: $51 \%$ versus 54\%). Higher percentages of individuals in CKD stages 3-5 for both eGFRscr and eGFRcys were taking antihypertensive medication (eGFRscr: $46 \%$ versus $25 \%$ and eGFRcys: $42 \%$ versus $22 \%$ ) and had a history of CVD (eGFRscr: $22 \%$ versus $7 \%$ and eGFRcys: $14 \%$ versus $5 \%$; Table 1).

Venular tortuosity was associated with an increased risk of CKD stages 3-5 characterised by eGFRscr in all models (fully adjusted odds ratio $[\mathrm{OR}]=1.30$; 95\% CI: $1.10,1.54 ; P<0.01$, respectively), although this was not the case for CKD stages $3-5$ defined by eGFRcys $(P>$ 
Table 1 Participant summary characteristics comparisons for CKD stages 1-2 and CKD stages 3-5

\begin{tabular}{|c|c|c|c|c|}
\hline Participant characteristic scr & All $(n=1860)$ & CKD stages $1-2(n=1713)$ & CKD stages 3-5 $(n=147)$ & $P$ Value \\
\hline Mean age (years, SD) & $62.0 \pm 8.5$ & $61.3 \pm 8.1$ & $70.5 \pm 9.0$ & $<0.01$ \\
\hline Female, n (\%) & $994(53.4)$ & $930(54.3)$ & $64(43.5)$ & 0.01 \\
\hline Education, primary level and below, $\mathrm{n}(\%)$ & $238(12.8)$ & $210(12.6)$ & $28(19.0)$ & 0.02 \\
\hline Diabetes, yes n (\%) & $429(23.1)$ & $380(22.2)$ & 49 (33.3) & $<0.01$ \\
\hline Mean BMI $\left(\mathrm{kg} / \mathrm{m}^{2}, \mathrm{SD}\right)$ & $28.6 \pm 5.0$ & $28.5 \pm 5.0$ & $29.2 \pm 4.4$ & 0.12 \\
\hline Mean systolic blood pressure (mmHg, SD) & $131.1 \pm 18$ & $131 \pm 18$ & $133 \pm 19$ & 0.27 \\
\hline Antihypertension medication, yes n (\%) & $506(27.2)$ & $438(25.6)$ & $68(46.3)$ & $<0.01$ \\
\hline Mean triglyceride (mmol/L, SD) & $1.7 \pm 1.0$ & $1.6 \pm 1.0$ & $1.8 \pm 0.8$ & 0.11 \\
\hline Mean HDL cholesterol (mmol/L, SD) & $1.6 \pm 0.5$ & $1.6 \pm 0.4$ & $1.5 \pm 0.5$ & $<0.01$ \\
\hline Mean LDL cholesterol (mmol/L, SD) & $3.5 \pm 1.1$ & $3.5 \pm 1.1$ & $3.1 \pm 1.2$ & $<0.01$ \\
\hline Cardiovascular disease, yes n (\%) & $143(7.7)$ & $111(6.5)$ & $32(21.8)$ & $<0.01$ \\
\hline Mean eGFR Creatinine (mL/min/1.73m², SD) & $82.2 \pm 14.9$ & $85.0 \pm 11.7$ & $49.6 \pm 7.8$ & $<0.01$ \\
\hline Participant characteristic cys & All $(n=1860)$ & CKD stages $1-2(n=1362)$ & CKD stages $3-5(n=498)$ & $P$ Value \\
\hline Mean age (years, SD) & $62.0 \pm 8.5$ & $59.8 \pm 7.4$ & $68.2 \pm 8.3$ & $<0.01$ \\
\hline Female, n (\%) & $994(53.4)$ & $739(54.3)$ & $255(51.2)$ & 0.24 \\
\hline Education, primary level and below, $\mathrm{n}(\%)$ & $238(12.8)$ & $138(10.1)$ & $100(20.1)$ & $<0.01$ \\
\hline Diabetes, yes n (\%) & $429(23.1)$ & $271(19.9)$ & $158(31.7)$ & $<0.01$ \\
\hline Mean BMI $\left(\mathrm{kg} / \mathrm{m}^{2}, \mathrm{SD}\right)$ & $28.6 \pm 5.0$ & $28.0 \pm 4.5$ & $30.3 \pm 5.6$ & $<0.01$ \\
\hline Mean systolic blood pressure (mmHg, SD) & $131.1 \pm 18$ & $130 \pm 19$ & $133 \pm 18$ & $<0.01$ \\
\hline Antihypertension medication, yes n (\%) & $506(27.2)$ & $297(21.8)$ & $209(42.0)$ & $<0.01$ \\
\hline Mean triglyceride (mmol/L, SD) & $1.7 \pm 1.0$ & $1.6 \pm 0.9$ & $1.8 \pm 1.0$ & $<0.01$ \\
\hline Mean HDL cholesterol (mmol/L, SD) & $1.6 \pm 0.5$ & $1.7 \pm 0.5$ & $1.6 \pm 0.4$ & $<0.01$ \\
\hline Mean LDL cholesterol (mmol/L, SD) & $3.5 \pm 1.1$ & $3.5 \pm 1.1$ & $3.2 \pm 1.2$ & $<0.01$ \\
\hline Cardiovascular disease, yes n (\%) & $143(7.7)$ & $73(5.4)$ & $70(14.1)$ & $<0.01$ \\
\hline Mean eGFR Cystatin C (mL/min/1.73m², SD) & $70.7 \pm 18.6$ & $78.6 \pm 14.7$ & $49.1 \pm 8.0$ & $<0.01$ \\
\hline
\end{tabular}

0.05; Table 2). Venular fractal dimension was associated with a reduced risk of CKD stages $3-5$ characterised by eGFRscr (OR = 0.84; 95\% CI: 0.70, 1.00; $P=0.05)$ but did not survive adjustment for the potential confounders age, sex, diabetes and smoking status, educational attainment, BMI, antihypertensive medication, CVD, SBP, triglycerides, $\mathrm{HDL}$ and LDL (OR $=0.87$; 95\% CI: 0.72, 1.05; $P=0.15)$. Arteriolar fractal dimension $(\mathrm{OR}=0.90 ; 95 \%$ CI: $0.82,1.00 ; P=0.05)$ and CRVE $(\mathrm{OR}=1.13$; 95\% CI: $1.02,1.25 ; P=0.02)$ were associated with risk of CKD stages 3-5 characterised by eGFRcys but again, did not survive adjustment for confounding factors. No further associations were detected between other RMPs and CKD stages 3-5 ( $P>0.05$; Table 2$)$.

In an unadjusted linear regression, $\operatorname{CRAE}(\beta$ [Beta] $=-$ 0.83; $95 \% \mathrm{CI}:-1.50,-0.15 ; P=0.02)$ and venular fractal dimension $(\beta=0.81 ; 95 \% \mathrm{CI}$ : $0.14,1.49 ; P=0.02)$ were associated with eGFRscr but did not survive adjustment for potential confounders in minimally or fully adjusted models $(P>0.05$; Table 3$)$. CRVE was associated with eGFRcys in an unadjusted linear regression model $(\beta=$ -1.23 ; 95\% CI: $-2.08,-0.38 ; P=<0.01$ ) but associations did not survive in the minimally adjusted or fully adjusted models. No further associations were detected between RMPs assessed as standardised Z-scores in a linear regression analysis with eGFRscr and eGFRcys $(P>0.05$; Table 3$)$.

\section{Discussion}

The eye provides an opportunistic non-invasive evaluation of the retinal microvasculature that may represent ongoing microvascular pathology elsewhere in the body. The majority of previously published studies have used measures of retinal vascular calibre (CRAE, CRVE and AVR), with only a small number evaluating other RMPs such as fractal dimension and tortuosity [27, 28]. Benitez-Aguirre and colleagues reported associations between venular tortuosity and incident renal dysfunction 
Table 2 Association of retinal vessel parameters and CKD status using binary logistic regression (scr and cys)

\begin{tabular}{|c|c|c|c|c|c|c|c|c|c|}
\hline \multirow{2}{*}{$\begin{array}{l}\text { CKD status scr } \\
\text { Retinal parameter }\end{array}$} & \multicolumn{3}{|c|}{ Unadjusted } & \multicolumn{3}{|c|}{ Minimally adjusted } & \multicolumn{3}{|c|}{ Fully adjusted } \\
\hline & OR & $95 \% \mathrm{Cl}$ & $P$ Value & OR & $95 \% \mathrm{Cl}$ & $P$ Value & OR & $95 \% \mathrm{Cl}$ & $P$ Value \\
\hline${ }^{\mathrm{a}}$ CRAE (PX) & 1.17 & $1.00,1.38$ & 0.06 & 1.12 & $0.94,1.34$ & 0.20 & 1.15 & $0.95,1.38$ & 0.14 \\
\hline${ }^{\mathrm{a}}$ CRVE (PX) & 1.10 & $0.93,1.30$ & 0.27 & 1.06 & $0.89,1.26$ & 0.54 & 1.03 & $0.86,1.23$ & 0.76 \\
\hline${ }^{\mathrm{a} A V R}$ & 1.06 & $0.89,1.25$ & 0.53 & 1.04 & $0.87,1.25$ & 0.64 & 1.09 & $0.91,1.30$ & 0.37 \\
\hline 'aFractal dimension arteriolar & 1.02 & $0.86,1.21$ & 0.83 & 1.10 & $0.91,1.32$ & 0.33 & 1.09 & $0.90,1.32$ & 0.36 \\
\hline${ }^{\mathrm{a} F r a c t a l}$ dimension venular & 0.86 & $0.73,1.00$ & 0.05 & 0.89 & $0.75,1.07$ & 0.22 & 0.87 & $0.72,1.05$ & 0.15 \\
\hline${ }^{\mathrm{ab}}$ Tortuosity arteriolar & 1.14 & $0.96,1.34$ & 0.14 & 1.13 & $0.94,1.35$ & 0.19 & 1.10 & $0.91,1.32$ & 0.33 \\
\hline${ }^{\mathrm{ab}}$ Tortuosity venular & 1.36 & $1.16,1.59$ & $<0.01$ & 1.34 & $1.14,1.59$ & $<0.01$ & 1.30 & $1.10,1.54$ & $<0.01$ \\
\hline CKD status cys & \multicolumn{3}{|c|}{ Unadjusted } & \multicolumn{3}{|c|}{ Minimally adjusted } & \multicolumn{3}{|c|}{ Fully adjusted } \\
\hline Retinal parameter & OR & $95 \% \mathrm{Cl}$ & $P$ Value & OR & $95 \% \mathrm{Cl}$ & $P$ Value & OR & $95 \% \mathrm{Cl}$ & $P$ Value \\
\hline${ }^{a}$ CRAE (PX) & 1.09 & $0.98,1.21$ & 0.10 & 1.05 & $0.94,1.18$ & 0.39 & 1.09 & $0.96,1.23$ & 0.19 \\
\hline${ }^{\mathrm{a} C R V E}(\mathrm{PX})$ & 1.13 & $1.02,1.25$ & 0.02 & 1.12 & $1.00,1.25$ & 0.06 & 1.08 & $0.96,1.22$ & 0.21 \\
\hline${ }^{\mathrm{a}} \mathrm{AVR}$ & 0.97 & $0.87,1.07$ & 0.55 & 0.94 & $0.84,1.06$ & 0.33 & 1.00 & $0.89,1.14$ & 0.97 \\
\hline 'a Fractal dimension arteriolar & 0.90 & $0.82,1.00$ & 0.05 & 0.95 & $0.84,1.06$ & 0.33 & 0.98 & $0.87,1.10$ & 0.69 \\
\hline${ }^{\mathrm{a}}$ Fractal dimension venular & 0.92 & $0.83,1.02$ & 0.10 & 0.98 & $0.87,1.10$ & 0.68 & 0.98 & $0.87,1.11$ & 0.77 \\
\hline abTortuosity arteriolar & 1.07 & $0.96,1.18$ & 0.23 & 1.06 & $0.94,1.18$ & 0.37 & 1.03 & $0.91,1.16$ & 0.66 \\
\hline${ }^{\mathrm{ab}}$ Tortuosity venular & 1.10 & $1.00,1.22$ & 0.06 & 1.08 & $0.97,1.21$ & 0.17 & 1.03 & $0.92,1.17$ & 0.59 \\
\hline
\end{tabular}

Abbreviations: eGFR Estimated glomerular filtration rate (Calculated using the CKD-EPI equation), CKD Chronic Kidney Disease, CRAE Central Retinal Arteriolar Equivalent, CRVE Central Retinal Venular Equivalent, AVR Retinal Arteriole/Venular Ratio, scr Serum Creatinine, cys Serum Cystatin C, CI Confidence Interval, OR

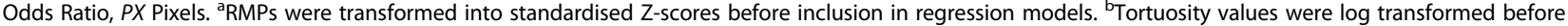
inclusion in regression models to produce normal distribution. Minimally adjusted models included age (yrs) and sex, with fully adjusted models also including diabetes and smoking status, cardiovascular disease, educational attainment, body mass index, antihypertensive medication, systolic blood pressure, triglycerides, high and low-density lipoproteins levels. $P$ values and $95 \%$ confidence intervals were generated from the regression models

in a prospective cohort of 511 adolescents with type 1 diabetes [29]. Our findings also identify increased venular tortuosity in association with CKD status characterised by eGFRscr $<60 \mathrm{~mL} / \mathrm{min} / 1.73 \mathrm{~m}^{2}$. Notably, the association was independent of a broad array of potential confounders including age, sex, diabetes and smoking status, educational attainment, CVD, BMI, antihypertensive medication, SBP, triglycerides, HDL and LDL levels $(\mathrm{OR}=1.30$; 95\% CI: $1.10,1.54 ; P<0.01)$. The number of individuals characterised with CKD stages $3-5$ based on eGFRscr, was considerably lower in contrast to those characterised by eGFRcys (147 versus 498, representing $8 \%$ or $27 \%$ of the study participants respectively, Table $1)$. The mechanisms that underlie venular tortuosity are unclear. Low levels of vessel tortuosity are not uncommon in the absence of overt clinical symptoms and high levels of vessel tortuosity have been reported in association with ischaemic changes in more distal organs [30]. Tortuous retinal vessels have been reported as the first identifiable vascular change in many retinopathies and in association with vascular disease, older age, lower HDL levels, hypertension, diabetes and genetic disorders such as hereditary vascular retinopathy and familial retinal arteriolar tortuosity [27-37]. Vessel tortuosity may result from mechanical instability under the influence of haemodynamic alterations that include endothelial dysfunction and blood flow leading to vessel remodelling [29]. Interestingly, the association between venular tortuosity and CKD stages 3-5 was strongest among the 147 participants with poorer renal function for both estimating equations compared to the 351 additional participants identified using the cystatin $\mathrm{C}$ equation only (mean eGFRscr: $49.6[n=147]$ versus $75.4[n=351] \mathrm{mL} /$ $\min / 1.73 \mathrm{~m}^{2}$ and eGFRcys: $45.1[n=147]$ versus 51.5 $[n=351] \mathrm{mL} / \mathrm{min} / 1.73 \mathrm{~m}^{2}$; data not shown). As such, retinal microvascular tortuosity may reflect changes in the kidney associated with CKD stages 3-5 but given the cross-sectional nature of this study, it is not possible to differentiate cause and effect [30,38].

Several cross-sectional studies have previously reported associations between RMPs and renal dysfunction $[16,18,39,40]$ including arteriolar narrowing in those with eGFR $<60 \mathrm{~mL} / \mathrm{min} / 1.73 \mathrm{~m}^{2}[41,42]$. In contrast, other studies have failed to detect associations between RMPs and renal function [43, 44]. We failed to detect any associations between CRAE and CKD stages 3-5 defined as eGFR $<60 \mathrm{~mL} / \mathrm{min} / 1.73 \mathrm{~m}^{2}$. A study by Edwards and colleagues hypothesised that abnormalities in the retinal microvasculature were associated with renal dysfunction in an elderly population. However, despite reported associations between retinopathy and renal function, no associations between retinal arteriolar or 
Table 3 Association of retinal vessel parameters and eGFR using linear regression (scr and cys)

\begin{tabular}{|c|c|c|c|c|c|c|c|c|c|}
\hline \multirow{2}{*}{$\begin{array}{l}\text { eGFR scr } \\
\text { Retinal parameter }\end{array}$} & \multicolumn{3}{|c|}{ Unadjusted } & \multicolumn{3}{|c|}{ Minimally adjusted } & \multicolumn{3}{|c|}{ Fully adjusted } \\
\hline & $\beta$ & $95 \% \mathrm{Cl}$ & $P$ Value & $\beta$ & $95 \% \mathrm{Cl}$ & $P$ Value & $\beta$ & $95 \% \mathrm{Cl}$ & $P$ Valu \\
\hline${ }^{\mathrm{a}}$ CRAE (PX) & -0.83 & $-1.50,-0.15$ & 0.02 & -0.47 & $-1.06,-.12$ & 0.12 & -0.54 & $-1.13,0.06$ & 0.08 \\
\hline${ }^{a}$ CRVE (PX) & -0.17 & $-0.84,0.51$ & 0.63 & 0.11 & $-0.48,0.70$ & 0.72 & 0.11 & $-0.48,0.69$ & 0.72 \\
\hline${ }^{\mathrm{a}} \mathrm{AVR}$ & -0.56 & $-1.24,0.12$ & 0.11 & -0.49 & $-1.08,0.10$ & 0.11 & -0.54 & $-1.13,0.05$ & 0.07 \\
\hline ªFractal dimension arteriolar & 0.27 & $-0.41,0.94$ & 0.44 & -0.14 & $-0.73,0.46$ & 0.65 & -0.03 & $-0.62,0.56$ & 0.92 \\
\hline aFractal dimension venular & 0.81 & $0.14,1.49$ & 0.02 & 0.35 & $-0.24,0.94$ & 0.25 & 0.41 & $-0.18,1.00$ & 0.17 \\
\hline ab Tortuosity arteriolar & -0.37 & $-1.05,0.31$ & 0.28 & -0.23 & $-0.82,0.36$ & 0.44 & -0.18 & $-0.78,0.40$ & 0.53 \\
\hline${ }^{\mathrm{ab}}$ Tortuosity venular & -0.47 & $-1.14,0.21$ & 0.18 & -0.27 & $-0.87,0.32$ & 0.36 & -0.16 & $-0.74,0.43$ & 0.59 \\
\hline eGFR cys & \multicolumn{3}{|c|}{ Unadjusted } & \multicolumn{3}{|c|}{ Minimally adjusted } & \multicolumn{3}{|c|}{ Fully adjusted } \\
\hline Retinal parameter & $\beta$ & $95 \% \mathrm{Cl}$ & $P$ Value & $\beta$ & $95 \% \mathrm{Cl}$ & $P$ Value & $\beta$ & $95 \% \mathrm{Cl}$ & $P$ Value \\
\hline${ }^{\mathrm{a} C R A E}(\mathrm{PX})$ & -0.68 & $-1.53,0.17$ & 0.12 & -0.34 & $-1.07,0.40$ & 0.37 & -0.46 & $-1.17,0.26$ & 0.21 \\
\hline${ }^{\mathrm{a}}$ CRVE (PX) & -1.23 & $-2.08,-0.38$ & $<0.01$ & -0.87 & $-1.60,-0.13$ & 0.02 & -0.53 & $-1.23,0.17$ & 0.14 \\
\hline${ }^{\mathrm{a}} \mathrm{AVR}$ & 0.55 & $-0.30,1.39$ & 0.21 & 0.54 & $-0.20,1.28$ & 0.15 & 0.12 & $-0.59,0.83$ & 0.73 \\
\hline${ }^{\mathrm{a} F}$ ractal dimension arteriolar & 0.55 & $-0.29,1.40$ & 0.20 & -0.04 & $-0.78,0.70$ & 0.92 & -0.15 & $-0.85,0.55$ & 0.68 \\
\hline${ }^{\mathrm{a}}$ Fractal dimension venular & 0.14 & $-0.71,0.99$ & 0.75 & -0.48 & $-1.22,0.26$ & 0.20 & -0.33 & $-1.03,0.37$ & 0.35 \\
\hline${ }^{\mathrm{ab}}$ Tortuosity arteriolar & -0.69 & $-1.53,0.16$ & 0.11 & -0.56 & $-1.29,0.18$ & 0.14 & -0.43 & $-1.13,0.27$ & 0.23 \\
\hline ab Tortuosity venular & -0.82 & $-1.67,0.03$ & 0.06 & -0.53 & $-1.26,0.21$ & 0.16 & -0.24 & $-0.94,0.45$ & 0.49 \\
\hline
\end{tabular}

Abbreviations: eGFR Estimated glomerular filtration rate (Calculated using the CKD-EPI equation), CRAE Central Retinal Arteriolar Equivalent, CRVE Central Retinal Venular Equivalent, AVR Retinal Arteriole/Venular Ratio, scr Serum Creatinine, cys Serum Cystatin C, Cl Confidence Interval, $\beta$ Beta, PX Pixels. ${ }^{\mathrm{a}} \mathrm{RMPs}$ were

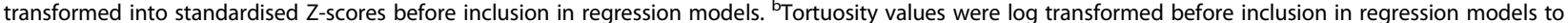
produce normal distribution. Minimally adjusted models included age (yrs) and sex, with fully adjusted models also including, diabetes and smoking status, cardiovascular disease, educational attainment, body mass index, antihypertensive medication, systolic blood pressure, triglycerides, high and low-density lipoproteins levels. P values and 95\% confidence intervals were generated from the regression models

venular calibre and renal function were detected [45]. Similarly, our study also failed to detect any association between RMPs and the continuous measure of eGFR (scr or cys) or the dichotomised variable of CKD status characterised by eGFRcys.

Our study had several limitations. Firstly, although not routinely measured in population-based studies, the absence of ACR limited the characterisation of renal function in this older population, which was reliant on single measures of scr and cys. Although single serum measures of scr or cys is common in population-based epidemiological studies it differs from clinical CKD staging which, in the absence of proteinuria, depends on two measures of eGFR $<60 \mathrm{~mL} / \mathrm{min} / 1.73 \mathrm{~m}^{2}$ at least 3 months apart [46]. Single eGFR measures sampled from population-based studies usually represent a reliable estimate in comparison to clinically confirmed cases, which can show greater variability. As such, population-based studies may not accurately reflect clinically observed eGFR and may not be directly comparable to clinically confirmed CKD. Secondly, the predominantly white study population aged greater than 50 yrs. may limit the generalisability of our findings to other populations. Thirdly, although NICOLA is a longitudinal study, data was only available for baseline measures facilitating analyses of cross-sectional associations between RMPs and renal function which are not indicative of the potential predictive capacity any associations may represent. Finally, although we adjusted for major potential confounders, the possibility of residual confounding by variables that were not included in the analyses remains.

Despite these limitations, our study had several strengths including the population-based design. NICOLA provided a robust study size with wellcharacterised individuals with a wide range of demographic factors and clinical variables including comorbidities and medications, enabling adjustment for potential confounding factors. In addition, the availability of optic disc centred retinal fundus images provides a more robust determination of RMPs using in silico tools in comparison to studies reliant on macula centred images, which are largely confined to evaluation of the microvasculature in the retinal temporal arcades. Furthermore, we used the CKD-EPI equation which is considered a more reliable measure of renal function, particularly at higher eGFR values [47]. Previous studies have largely defined eGFR based on scr to characterise renal function. We used both scr and cys to estimate GFR and characterise CKD status. Previous studies have reported variation in the characterisation of renal function when using eGFRcys or eGFRscr CKD classification [24, 48-50]. Husain and colleagues compared eGFRcys 
(CKD-EPI 2012 equation) and eGFRscr (CKD-EPI 2009 equation) to classify CKD status in an elderly cohort. They reported a lower mean eGFRcys of $23 \pm 15 \mathrm{~mL} /$ $\mathrm{min} / 1.73 \mathrm{~m}^{2}$ compared to eGFRscr, leading to a higher classification of CKD prevalence ( $71 \%$ vs $22 \%$ respectively, $P=<0.001 ; 49)$. On average, the mean eGFRcys for all participants in the current study was $11.5 \mathrm{~mL} / \mathrm{min} /$ $1.73 \mathrm{~m}^{2}$ lower than the mean eGFRscr, and $0.5 \mathrm{~mL} / \mathrm{min} /$ $1.73 \mathrm{~m}^{2}$ lower for those with CKD stages $3-5$ and 6.4 $\mathrm{mL} / \mathrm{min} / 1.73 \mathrm{~m}^{2}$ lower for those classified with CKD stages $1-2$, representing a mean greater difference in those with better renal function. Several advantages have been proposed for the use of cystatin $\mathrm{C}$ over serum creatinine as a more sensitive estimate of renal function. Cystatin $\mathrm{C}$ is released by all cell types, freely filtered by the kidney and is less confounded by diet, ethnicity, muscle mass or sex [51]. Lees and colleagues (2019) demonstrated improved sensitivity of cystatin C-based eGFR for the prediction of all-cause mortality and fatal/ nonfatal CVD in 440,526 participants of the UK Biobank study [51]. As such, we provide data that examines associations using both eGFR determinants of renal function for comparative purposes.

Retinal imaging offers a novel opportunity to complement CKD screening in different clinical and public health settings. Individuals with CKD typically remain asymptomatic for a prolonged period (years) and appropriate screening would facilitate targeted implementation of preventive measures to reduce health-care burden. Moreover, CKD awareness is as low as $10 \%$ and studies to identify the potential benefits and risks of screening, screening measures, and target groups for screening of asymptomatic individuals, would help inform inconsistent screening guidelines that exist across professional bodies [52]. Advances in retinal fundus imaging technology and integration with machine learning approaches will enable rapid, non-invasive, point-of-care diagnoses, which may enhance screening service provision and improved screening compliance [53]. Retinal cameras are common in primary care settings and high street opticians for diabetic retinopathy screening. Recent advances in smartphone technology, combined with the utility of machine learning approaches, highlights the feasibility and potential offered by non-invasive retinal photography as an adjunctive or opportunistic screening tool for CKD in the community [53].

\section{Conclusions}

In summary, our findings identify increased variation in retinal venular tortuosity in association with poorer renal function (specifically CKD status characterised by scr) in an older population. These non-invasive retinal measures may help identify mechanistic pathways of microvascular variation early in the disease process in individuals at increased risk of CKD stages 3-5. Identification of such individuals may offer clinical utility for the stratification of those most in need of more frequent surveillance and earlier therapeutic intervention to limit the extent of disease progression.

\section{Supplementary information}

Supplementary information accompanies this paper at https://doi.org/10. 1186/s12882-020-02031-0.

Additional file 1: Supplementary Table 1. Comparison of

demographic characteristics between all participants with retinal fundus imaging with and without VAMPIRE retinal measures. Supplementary

Table 2. STROBE Statement-Checklist of items that should be included in reports of cross-sectional studies. Supplementary Figure 1.

Additional file 2:. Supplementary file 1.

\section{Abbreviations}

NICOLA: The Northern Ireland Cohort for Longitudinal Ageing; CKD: Chronic kidney disease; CKD-EPI: Chronic Kidney Disease Epidemiology Collaboration eq.; BMI: Body Mass Index; eGFR: Estimated glomerular filtration rate; scr: Serum Creatinine; cys: Serum Cystatin C; HDL: High-density Lipoprotein; LDL: Low-density Lipoprotein; CRAE: Central Retinal Arteriolar Equivalent; CRVE: Central Retinal Venular Equivalent; AVR: Retinal Arteriole/Venular Ratio; SD: Standard deviation; PX: Pixels; eGFRscr: Estimated glomerular filtration rate from serum creatinine; eGFRcys: Estimated glomerular filtration rate from serum cystatin C; NI: Northern Ireland; CPH: Centre for Public Health; SBP: Systolic blood pressure; DBP: Diastolic blood pressure; Cl: Confidence intervals; CKD scr: Chronic kidney disease classification from serum creatinine CKD cys: Chronic kidney disease classification from serum cystatin C; $\beta$ : Beta value; $\mu \mathrm{m}$ : Microns; ICC's: Intraclass correlation coefficients; VAMPIRE: Vessel Assessment and Measurement Platform for Images of the Retina; MABP: Mean arteriole blood pressure; RMPs: Retinal microvascular parameters; Yrs: Years; ACR: Urine albumin to creatinine ratio; CVD: Cardiovascular disease; SBP: Systolic blood pressure

\section{Acknowledgements}

We are grateful to all the participants of the NICOLA Study, and the whole NICOLA team, which includes nursing staff, research scientists, clerical staff, computer and laboratory technicians, managers and receptionists. The Atlantic Philanthropies, the Economic and Social Research Council, the UKCRC Centre of Excellence for Public Health Northern Ireland, the Centre for Ageing Research and Development in Ireland, the Office of the First Minister and Deputy First Minister, the Health and Social Care Research and Development Division of the Public Health Agency, the Wellcome Trust/ Wolfson Foundation and Queen's University Belfast provide core financial support for NICOLA. The authors alone are responsible for the interpretation of the data and any views or opinions presented are solely those of the authors and do not necessarily represent those of the NICOLA Study team.

\section{Authors' contributions}

RON undertook retinal measurements, data analysis and was a major contributor to the writing of the manuscript. GMK and APM proposed the research hypothesis and were major contributors to the writing of the manuscript. FK and IY conceived and designed the NICOLA. BMG acquired and interpreted NICOLA health assessment data. $\mathrm{RH}$ acquired and interpreted NICOLA ophthalmic data. All authors revised the manuscript critically for important intellectual content; and agreed to be accountable for all aspects of the work in ensuring that questions related to the accuracy or integrity of any part of the work are appropriately investigated and resolved. Finally all authors read and approved the final manuscript before submission.

\section{Funding}

Rachael O'Neill was supported by a PhD studentship from the Department for Economy, Northern Ireland.

This work was supported by the following funders who provide core financial support for the NICOLA Study: the Atlantic Philanthropies; the Economic and Social Research Council; the UKCRC Centre of Excellence for 
Public Health Northern Ireland; the Centre for Ageing Research and Development in Ireland; the Office of the First Minister and Deputy First Minister; the Health and Social Care Research and Development Division of the Public Health Agency; the Wellcome Trust/Wolfson Foundation; and Queen's University Belfast.

\section{Availability of data and materials}

The data that support the findings of this study are available from the Northern Ireland Cohort of Longitudinal Ageing but restrictions apply to the availability of this data, which was used under license for the current study, and so is not publicly available. Data may however available from the corresponding authors upon reasonable request and provided there is permission from NICOLA.

\section{Ethics approval and consent to participate}

Written informed consent was obtained from participants prior to taking part following ethical approval from the Queen's University Belfast School Research Ethics Committee in compliance with the Declaration of Helsinki (SREC 12/23).

\section{Consent for publication}

Not applicable.

\section{Competing interests}

The authors declare that they have no competing interests.

\section{Received: 19 December 2019 Accepted: 20 August 2020}

Published online: 03 September 2020

\section{References}

1. Couser WG, Remuzzi G, Mendis S, Tonelli M. The contribution of chronic kidney disease to the global burden of major non-communicable diseases. Kidney Int. 2011;80(12):1258-70.

2. Zhou XJ, Rakheja D, Yu X, Saxena R, Varizi ND, Silva FG. The aging kidney. Kidney Int. 2008:74:710-20.

3. Benito MH, Fernandez-Reyes MJ, Sanchez R. Implications outcome of chronic kidney disease in elderly. Nefrologia. 2010;30:151-7.

4. Kerr M, Bray B, Medcalf J, O'Donoghue DJ, Matthews B. Cost: estimating the financial cost of chronic kidney disease to the NHS in England. Nephrol Dial Transplant. 2012:27(3):73-80.

5. National Kidney Foundation. K/DOQI Clinical practice guidelines for chronic kidney disease: evaluation, classification, and stratification. Am J Kidney Dis. 2002;39:1-266

6. Stevens LA, Levey AS. Status and future perspectives for CKD testing. Am J Kidney Dis. 2009;53(3):17-26.

7. Futrakul N, Butthep P, Futrakul P. Altered vascular homeostasis in chronic kidney disease. Clin Hemorheol Microcirc. 2008;38(3):201-7.

8. Matsushita K, Van der Velde M, Astor BC, Woodward M, Levey AS, De Jong $P E$, et al. Association of estimated glomerular filtration rate and albuminuria with all-cause and cardiovascular mortality in general population cohorts: a collaborative meta-analysis. Lancet. 2010;375(9731):2073-81.

9. Foreman KJ, Marquez N, Dolgert A, Fukutaki K, Fullman N, McGaughey M, et al. Forecasting life expectancy, years of life lost, and all-cause and causespecific mortality for 250 causes of death: reference and alternative scenarios for 2016-40 for 195 countries and territories. Lancet. 2018; 392(10159):2052-90.

10. Tonelli M, Riella M. Chronic kidney disease and the ageing population. Indian J Nephrol. 2014;24(2):71-4.

11. Jha V, Garcia-Garcia G, Iseki K, Li Z, Naicker S, Plattner B, et al. Chronic kidney disease: global dimension and perspectives. Lancet. 2013;382(9888): 260-72.

12. Blum M, Saemann A, Wolf G. The eye, the kidney and microcirculation. Nephrol Dial Transplant. 2010;26(1):4-6.

13. Awua-Larbi S, Wong TY, Cotch MF, Durazo-Arvizu R, Jacobs DR Jr. Retinal arteriolar caliber and urine albumin excretion: the multi-ethnic study of atherosclerosis. Nephrol Dial Transplant. 2011:26:3523-8.

14. Lim LS, Cheung CY, Sabanayagam C, Lim SC, Tai ES, Huang L, et al. Structural changes in the retinal microvasculature and renal function. Invest Ophthalmol Vis Sci. 2013;54:2970-6
15. Sabanayagam C, Tai ES, Shankar A, Lee J, Sun C, Wong TY. Retinal arteriolar narrowing increases the likelihood of chronic kidney disease in hypertension. Int J Hypertens. 2009;27:2209-17.

16. Yip W, Guan Ong P, Teo BW, Cheung CY, Tai ES, Cheng CY, et al. Retinal vascular imaging markers and incident chronic kidney disease: a prospective cohort study. Sci Rep. 2017:7:9374.

17. Evans RD, Rosner M. Ocular abnormalities associated with advanced kidney disease and hemodialysis. Semin Dial. 2005;18:252-7.

18. Wong TY, Coresh J, Klein R, Muntner P, Couper DJ, Sharrett AR, et al. Retinal microvascular abnormalities and renal dysfunction: the atherosclerosis risk in communities' study. J Am Soc Nephrol. 2004;15(9):2469-76.

19. Wong CW, Wong TY, Cheng C-Y, et al. Kidney and eye diseases: common risk factors, etiological mechanisms, and pathways. Kidney Int. 2014;85(6): 1290-302.

20. Patel DV, Snead MP, Satchi K. Retinal arteriolar calcification in a patient with chronic renal failure. Brit J Ophthalmol. 2002;86:1063.

21. Gramatikov BI. Modern technologies for retinal scanning and imaging: an introduction for the biomedical engineer. Biomed Eng. 2014;13:52.

22. McCann P, Hogg R, Wright DM, Pose-Bazarra S, Chakravarthy U, Peto T, et al. Glaucoma in the Northern Ireland Cohort for the Longitudinal Study of Ageing (NICOLA): cohort profile, prevalence, awareness and associations [published online ahead of print, $2020 \mathrm{Feb} 7$ ]. Br J Ophthalmol. 2020; bjophthalmol-2019-315330. https://doi.org/10.1136/bjophthalmol-2019315330

23. Levey AS, Stevens LA, Schmid CH, Zhang YL, Castro AF 3rd, Feldman HI, et al. A new equation to estimate glomerular filtration rate. Ann Intern Med. 2009;150(9):604-12.

24. Inker $\mathrm{LA}$, Schmid $\mathrm{CH}$, Tighiouart $\mathrm{H}$, Eckfeldt $\mathrm{JH}$, Feldman $\mathrm{HI}$, Greene T, et al. Estimating glomerular filtration rate from serum creatinine and cystatin C. N Engl J Med. 2012:367(1):20-9.

25. Fetit AE, Doney AS, Hogg S, Wang R, MacGillivray T, Wardlaw JM, et al. A multimodal approach to cardiovascular risk stratification in patients with type 2 diabetes incorporating retinal, genomic and clinical features. Sci Rep. 2019;9(1):3591

26. MacGillivray TJ, Cameron JR, Zhang Q, El-Medany A, Mulholland C, Sheng Z, et al. Suitability of UK biobank retinal images for automatic analysis of morphometric properties of the vasculature. PLoS One. 2015;10(5):e0127914.

27. Pries AR, Secomb TW. Modelling structural adaptation of microcirculation. Microcirculation. 2008;15:753-64.

28. Ikram MK, Cheung CY, Lorenzi M, Klein R, Jones TLZ, Wong TY. Retinal vascular caliber as a biomarker for diabetes microvascular complications. Diabetes Care. 2013:36(3):750-9.

29. Benitez-Aguirre PZ, Sasongko MB, Craig ME, Jenkins AJ, Cusumano J, Cheung $\mathrm{N}$, et al. Retinal vascular geometry predicts incident renal dysfunction in young people with type 1 diabetes. Diabetes Care. 2012;35: 599-604.

30. Han HC. Twisted blood vessels: symptoms, etiology and biomechanical mechanisms. J Vasc Res. 2012;49:185-97.

31. Amemiya T, Bhutto IA. Retinal vascular changes and systemic diseases: corrosion cast demonstration. Ital J Anat Embryol. 2001;106:237-44.

32. Hughes AD, Martinez-Perez E, Jabbar AS, Hassan A, Witt NW, Mistry PD, et al. Quantification of topological changes in retinal vascular architecture in essential and malignant hypertension. Int J Hypertens. 2006;24:889-94

33. Taarnhoj NC, Munch IC, Sander B, Kessel L, Hougaard JL, Kyvik K, et al. Straight versus tortuous retinal arteries in relation to blood pressure and genetics. Brit J Ophthalmol. 2008;92:1055-60.

34. Sutter FK, Helbig H. Familial retinal arteriolar tortuosity: a review. Surv Ophthalmol. 2003;48(3):245-55.

35. Vahedi K, Massin P, Guichard JP, Miocque S, Polivka M, Goutieres F, et al. Hereditary infantile hemiparesis, retinal arteriolar tortuosity and leukoencephalopathy. Neurology. 2003;60(1):57-63.

36. Kylstra JA, Wierzbicki T, Wolbarsht ML, Landers MB 3rd, Stefansson E. The relationship between retinal vessel tortuosity, diameter, and transmural pressure. Graefes Arch Clin Exp Ophthalmol. 1986;224:477-80

37. Bhutto IA, Amemiya T. Vascular changes in retinas of spontaneously hypertensive rats demonstrated by corrosion casts. Ophthalmic Res. 1997;29: $12-23$.

38. Cheung CYL, Zheng Y, Hsu W, Lee ML, Lau OP, Mitchell P, et al. Retinal vascular tortuosity, blood pressure and cardiovascular risk factors. Ophthalmology. 2011;118(5):812-8. 
39. Yau JW, Xie J, Kawasaki R, Kramer H, Shlipak M, Klein R, et al. Retinal arteriolar narrowing and subsequent development of CKD stage 3: the multi-ethnic study of atherosclerosis (MESA). Am J Kidney Dis. 2011;58:39-46.

40. Grunwald JE, Alexander J, Ying GS, Maguire M, Daniel E, Whittock-Martin R, et al. Retinopathy and chronic kidney disease in the chronic renal insufficiency cohort (CRIC) study. Arch Ophthalmol. 2012;130(9):1136-44.

41. Sabanayagam C, Shankar A, Koh D, Chia KS, Saw SM, Lim SC, et al. Retinal microvascular caliber and chronic kidney disease in an Asian population. Am J Epidemiol. 2009;169(5):625-32.

42. Baumann M, Schwarz S, Kotliar K, Von Eynatten M, Trucksaess AS, Burjhardt $K$, et al. Non-diabetic chronic kidney disease influences retinal microvasculature. Kidney Blood Press Res. 2009;32(6):428-33.

43. McGowan A, Silvestri G, Moore E, Silvestri V, Patterson C, Maxwell AP, et al. Evaluation of the retinal vasculature in hypertension and chronic kidney disease in an elderly population of Irish nuns. PLoS One. 2015:10(9):1-14.

44. McKay GJ, Paterson EN, Maxwell AP, Cardwell CC, Wang R, Hogg S, et al. Retinal microvascular parameters are not associated with reduced renal function in a study of individuals with type 2 diabetes. Sci Rep. 2018;8(1):3931.

45. Edwards MS, Wilson DB, Craven TE, Stafford J, Fried LF, Wong TY, et al. Associations between retinal microvascular abnormalities and declining renal function in the elderly population: the cardiovascular health study. Am J Kidney Dis. 2005:46(2):214-24.

46. Eknoyan G, Lameire N, Eckardt K. KDIGO 2012 clinical practice guideline for the evaluation and Management of Chronic Kidney Disease. Kidney Int. 2012;2:1.

47. Wu B, Bell K, Stanford A, Kern D, Tunceli O, Vupputuri S, et al. Understanding CKD among patients with T2DM: prevalence, temporal trends, and treatment patterns-NHANES 2007-2012. BMJ Open Diabetes Res Care. 2016;4(1):e000154

48. Husain SA, Willey JZ, Park Moon Y, Elkind MSV, Sacco RL, Wolf M, et al. Creatinine- versus cystatin C-based renal function assessment in the northern Manhattan study. PLoS One. 2018;13(11):e0206839.

49. Salminen $\mathrm{M}$, Laine $\mathrm{K}$, Korhonen $\mathrm{P}$, Wasen $\mathrm{E}$, Vahlberg $\mathrm{T}$, Isoaho $\mathrm{R}$, et al. Biomarkers of kidney function and prediction of death from cardiovascular and other causes in the elderly: a 9-year follow-up study. Eur J Intern Med. 2016;33:98-101.

50. Krolewski AS, Warram JH, Forsblom C, Smiles AM, Thorn L, Skupien J, et al. Serum concentration of cystatin $C$ and risk of end-stage renal disease in diabetes. Diabetes Care. 2012:35(11):2311-6.

51. Lees JS, Welsh CE, Celis-Morales CA, et al. Glomerular filtration rate by differing measures, albuminuria and prediction of cardiovascular disease, mortality and end-stage kidney disease. Nat Med. 2019;25(11):1753-60

52. Saunders MR, Cifu A, Vela M. Screening for chronic kidney disease. JAMA. 2015;314(6):615-6

53. Sabanayagam C, Xu D, Ting DSW, Nusinovici S, Banu R, Hamzah H, et al. A deep learning algorithm to detect chronic kidney disease from retinal photographs in community-based population. Lancet. 2020;7500(20):30063-7.

\section{Publisher's Note}

Springer Nature remains neutral with regard to jurisdictional claims in published maps and institutional affiliations.

\section{Ready to submit your research? Choose BMC and benefit from:}

- fast, convenient online submission

- thorough peer review by experienced researchers in your field

- rapid publication on acceptance

- support for research data, including large and complex data types

- gold Open Access which fosters wider collaboration and increased citations

- maximum visibility for your research: over $100 \mathrm{M}$ website views per year

At $\mathrm{BMC}$, research is always in progress.

Learn more biomedcentral.com/submissions 